\title{
Functionalizing Electrospun Fibers with Biologically Relevant Macromolecules
}

\author{
Cheryl L. Casper, Nori Yamaguchi, Kristi L. Kiick, and John F. Rabolt ${ }^{\star}$ \\ Department of Materials Science and Engineering, University of Delaware, Newark, Delaware \\ 19716, and Delaware Biotechnology Institute, 15 Innovation Way, Newark, Delaware 19711
}

\begin{abstract}
The development of functionalized polymers that can elicit specific biological responses is of great interest in the biomedical community, as well as the development of methods to fabricate these biologically functionalized polymers. For example, the generation of fibrous matrices with biological properties and fiber diameters commensurate with those of the natural extracellular matrix (ECM) may permit the development of novel materials for use in wound healing or tissue engineering. The goal of this work is, therefore, to create a biologically active functionalized electrospun matrix to permit immobilization and long-term delivery of growth factors. In this work, poly(ethylene glycol) functionalized with low molecular weight heparin (PEG-LMWH) was fabricated into fibers for possible use in drug delivery, tissue engineering, or wound repair applications. Electrospinning was chosen to process the LMWH into fiber form due to the small fiber diameters and high degree of porosity that can be obtained relatively quickly and using small amounts of starting material. Both free LMWH and PEG-LMWH were investigated for their ability to be incorporated into electrospun fibers. Each of the samples were mixed with a carrier polymer consisting of either a $10 \mathrm{wt} \%$ poly (ethylene oxide) (PEO) or $45 \mathrm{wt} \%$ poly(lactide-co-glycolide) (PLGA). Field emission scanning electron microscopy (FESEM), energy-dispersive X-ray analysis (EDX), UV-vis spectroscopy, and multiphoton microscopy were used to characterize the electrospun matrices. The incorporation of heparin into the electrospun PEO and PLGA fibers did not affect the surface morphology or fiber diameters. The fibers produced had diameters ranging from approximately 100 to $400 \mathrm{~nm}$. Toluidine blue assays of heparin suggest that it can be incorporated into an electrospun matrix at concentrations ranging from 3.5 to $85 \mu \mathrm{g}$ per milligram of electrospun fibers. Multiphoton microscopy confirmed that incorporation of PEG-LMWH into the matrix permits retention of the heparin for at least 14 days. Improvements in the binding of basic fibroblast growth factor to the electrospun fibers were also observed for fibers functionalized with PEG-LMWH over those functionalized with LMWH alone. The combination of these results suggests the utility for producing electrospun fibers that are appropriately functionalized for use in biomaterials applications.
\end{abstract}

\section{Introduction}

Increased interest in nanotechnology has made electrospinning an attractive approach as a polymer processing technique. The control of fiber diameter, porosity, and fiber surface morphology makes electrospun fibers useful in a range of applications including filtration, electronic, and biomedical applications. Electrospun fibers are ideal for use in biomedical applications such as tissue engineering and drug delivery due to the three-dimensional

*To whom correspondence should be addressed. Address: 201 Dupont Hall, University of Delaware, Newark, DE, 19716. Tel: 302-831-4476. Fax: 302-831-4545. E-mail: rabolt@udel.edu.

Supporting Information Available. Experimental details and results. This material is available free of charge via the Internet at http://pubs.acs.org. 
nanometer scale matrix that can be quickly produced using small quantities of starting material $(<50 \mathrm{mg})^{1-11}$ and then scaled up to form large mats and structures. ${ }^{12,13}$ Collagen, ${ }^{7}$ fibrinogen, 8 chitosan, ${ }^{14}$ poly(lactic acid), ${ }^{11}$ poly(L-lactide- $c o$ - $\varepsilon$-caprolactone), ${ }^{9}$ and poly(D,L-lactide-coglycolide $)^{5}$ are just a few of the polymers being investigated for use in electrospun drug delivery and tissue engineering constructs due to their biocompatibility. Electrospun fibers mimic the size scale of fibrous proteins found in the extracellular matrix $(50-150 \mathrm{~nm})^{8}$ and the threedimensional nature of the matrix allows for cells to infiltrate the matrix and proliferate. ${ }^{15}$ The fiber diameter alone, however, is not the only important role in creating an ECM-mimetic scaffold. The combination of small fiber diameters and fiber functionalization with PEGLMWH may provide functional benefits over other synthetic polymer fibers, given that the incorporation of heparin in synthetic polymeric hydrogel materials maintains bioactivity of growth factors. ${ }^{16-18}$.

When constructing electrospun fibers for biomedical applications, certain material considerations must be acknowledged. Some minimal properties of a drug delivery system are that the carrier polymer must be nonimmunogenic, degradable with nontoxic products, able to be excreted from the body, and deliver therapeutic molecules in a controlled and predictable manner. ${ }^{19}$ Manufacturing time and complexity, along with handling, must also be considered. ${ }^{19}$ In compliance with these requirements, PEO and PLGA serve as excellent candidates for fabrication of the matrix via electrospinning; indeed, the successful use of these polymers in a variety of tissue engineering and drug delivery applications has been well documented. ${ }^{20-}$ 26.

The choice of heparin for inclusion into an electrospun matrix was based on specific considerations. Heparin is a highly sulfated glycosaminoglycan that binds growth factors such as fibroblast growth factor (FGF), vascular endothelial growth factor (VEGF), heparin-binding epidermal growth factor (HBEGF), transforming growth factor- $\beta$ (TGF- $\beta$ ), and others; growth factors are important for controlling cellular activities such as cell growth and proliferation. 27-29 The binding of growth factors to glycosaminoglycans in the ECM is essential for storage, release, and preservation of the bioactivity of the growth factor. ${ }^{16,17,27,28,30}$ Heparin primarily serves to immobilize growth factors until release but is also known to control their mitogenic activity, and in several cases, serves as a cofactor to promote binding of the growth factors to their receptors. ${ }^{18}$ It has also been shown to regulate the growth of fibroblast and endothelial cells when combined with heparin-binding growth factors. ${ }^{27}$ For example, heparin incorporated into a chitosan hydrogel was found to induce neovascularization in vivo and to enhance the activity of acidic fibroblast growth factor (aFGF) and basic fibroblast growth factor (bFGF) and stabilize the growth factors in vitro. ${ }^{18} \mathrm{~A}$ variety of other reports also confirm the benefits of including heparin in a bulk hydrogel matrix ${ }^{16-18,31}$ and the extension to a fibrous matrix may provide additional structural advantages for producing materials that mimic the ECM. The incorporation of heparin into fiber form may allow for advantages that are not seen in hydrogel systems. Hydrogels have been shown, in some cases, to exhibit a lack of mechanical strength, ${ }^{23}$ rapid diffusion of encapsulated molecules, ${ }^{23}$ and some require cross-linking methods that are toxic to cells. ${ }^{32}$ The incorporation of molecules, such as heparin, into an electrospun matrix would allow exploitation of heparin's affinity for growth factors as a strategy to deliver them from a fibrous ECM-mimetic matrix while avoiding some of the previously mentioned issues with current delivery systems. Although there have been reports of the use of electrospun fibers as scaffolds for select drug delivery and biomaterials applications, the incorporation of bioactive molecules has not been widely exploited. Accordingly, the goal of this work was to fabricate functionalized electrospun fibers to produce a biologically active matrix that would allow the binding and delivery of growth factors for possible drug delivery applications. ${ }^{33}$ A four-arm star PEG with covalently attached LMWH was investigated for its capacity to be incorporated into electrospun fibers of PEO or PLGA and its effect on growth factor binding when compared to free LMWH. 


\section{Experimental Section}

\section{Materials and Methods}

Hydroxy terminated and thiol terminated four-arm star poly(ethylene glycol)s $\left(M_{\mathrm{n}}=10300\right.$; $M_{\mathrm{w}}=11300 \mathrm{~g} / \mathrm{mol}$ and $M_{\mathrm{n}}=10000 ; M_{\mathrm{w}}=10800 \mathrm{~g} / \mathrm{mol}$, respectively) were purchased from Polymer Source (Dorval, QC, Canada). LMWH sodium salt (porcine intestinal mucosa, avg. molecular weight $3000 \mathrm{~g} / \mathrm{mol}$ ) was obtained from Sigma Aldrich (St. Louis, MO). Alexa Fluor 350 carboxylic acid, succinimidyl ester was obtained from Molecular Probes (Eugene, OR).

Triethylamine was distilled from $\mathrm{CaH}_{2}$. The deionized water was generated from a Barnstead Diamond water purification system (Barnstead, Dubuque, IA, 18.1 M $\Omega$-cm resistivity). All other reagents were used as received. ${ }^{1} \mathrm{H}$ NMR spectra were acquired on a Bruker DRX-400 NMR spectrometer. The samples were prepared by dissolving $10 \mathrm{mg}$ of sample in $1 \mathrm{~mL}$ of dimethyl sulfoxide- $d_{6}$ (DMSO- $d_{6}$ ) or deuterium oxide. The resulting solutions were placed in $5 \mathrm{~mm}$ NMR tubes and the spectra were recorded under standard quantitative conditions at ambient temperature. All spectra recorded in deuterium oxide were referenced to sodium 2,2dimethyl-2-silapentane-5-sulfonate (DSS), which was added to each NMR tube prior to analysis.

Low molecular weight heparin $\left(M_{\mathrm{r}}=3000 \mathrm{~g} / \mathrm{mol}\right)$ was chosen because the reaction of maleimide functionalized high molecular weight heparin $\left(M_{\mathrm{r}}=12000\right)$ with the thiol functionalized four-arm star PEG would lead to a cross-linked network owing to the reaction of multiple PEG chain ends per heparin molecule. As $N$-deacetylated LMWH is estimated to have less than one randomly positioned free amino group per chain, ${ }^{34}$ the use of LMWH would not lead to a cross-linked network when reacted with the star PEG. The LMWH has been suggested to bind to growth factors, so the motivation behind the use of the LMWH was to produce soluble macromolecules that could be easily incorporated into fibers and still bind growth factors.

Synthesis of PEG-LMWH-Dye Conjugate-The details of the general synthesis of the PEG-LMWH conjugate are detailed elsewhere. ${ }^{34}$ The general description of the synthetic protocols for producing the PEG-LMWH-dye conjugate employed in this manuscript is briefly described below and is detailed in the Supporting Information.

\section{Synthesis of Maleimide and Alexa Fluor 350 Functionalized LMWH-N-}

deacetylated LMWH was neutralized via treatment with dry dowex 50WX4-200 resin (Sigma Aldrich, St. Louis, MO) and dissolved in anhydrous dimethylformamide (DMF). After filtration of the resin and addition of a few drops of triethylamine, the deacetylated LMWH was reacted with 4-( $\mathrm{N}$-maleimidomethyl)cyclo-hexanecarboxylic acid $\mathrm{N}$-hydroxysuccinimide ester (0.75 equiv.) (Sigma Aldrich, St. Louis, MO), followed by reaction with Alexa Fluor 350 carboxylic acid, succinimidyl ester (1 equiv.). The resulting product was purified via size exclusion chromatography employing a Superdex 200 gel filtration column (Amersham Biosciences, Piscataway, NJ) using $0.2 \mathrm{M} \mathrm{NaCl}$ at $0.5 \mathrm{~mL} / \mathrm{min}$ as eluting buffer. The heparincontaining fractions were combined and dialyzed against a SpectraPor membrane (MWCO $1000 \mathrm{Da}$ ) first with $1 \mathrm{M} \mathrm{NaCl}$ and finally with water (each dialysis bath was changed four times after 2, 8, 16, and $24 \mathrm{~h}$ ) and were then lyophilized to afford the final product. NMR characterization indicated maleimide and dye functionalization of the LMWH.

Synthesis of PEG-LMWH-Dye-The maleimide- and dye-functionalized LMWH was reacted with thiol-termined, four-arm star PEG $\left(M_{\mathrm{w}}=10000 \mathrm{~g} / \mathrm{mol}\right.$; Polymer Source, Dorval, Quebec) in degassed PBS ( $\mathrm{pH} 6.5,0.10 \mathrm{M}, 0.15 \mathrm{M} \mathrm{NaCl}$ ). Analysis of the reaction with Ellman's reagent indicated $96 \%$ consumption of the thiol. The reaction was quenched via addition of $N$-ethylmaleimide, and the product was purified via size exclusion chromatography 
as described above. NMR analysis of the resulting product indicates $73 \%$ functionalization of the star PEG with dye-modified LMWH (see the Supporting Information).

\section{Electrospinning and Characterization}

Fiber Spinning-The electrospinning apparatus consisted of a syringe pump (Orion Sage, Fisher, Fair Lawn, NJ), high voltage supply (Glassman Series EH, High Bridge, NJ), $1 \mathrm{~mL}$ syringe (Popper \& Sons, New Hyde Park, NY), and a needle (Hamilton, Reno, NV) with an inner diameter of $0.51 \mathrm{~mm}$. Electrospinning protocols were optimized prior to conditions chosen. A voltage of $-12 \mathrm{kV}$ was applied to the tip of the needle. Initially, $+12 \mathrm{kV}$ voltage was investigated for these studies but failed to electrospin. We believe the highly negatively charged solutions were attracted to the positive charge on the needle, therefore inhibiting ejection from the needle. Thus, a voltage of $-12 \mathrm{kV}$ was used for all studies. A grounded metal sheet was used as the target and was positioned $15 \mathrm{~cm}$ from the tip of the needle for sample collection. The concentration of the polymeric solutions varied depending on the carrier polymer employed. PEO (BDH Chemicals, Poole, U.K., $M_{\mathrm{w}}=300000 \mathrm{~g} / \mathrm{mol}$ ) was used at a $10 \mathrm{wt} \%$ concentration in water and a flow rate of $0.07 \mathrm{~mL} / \mathrm{min}$ was employed. PLGA (Sigma Aldrich, St. Louis, MO, 75:25, $M_{\mathrm{w}}=90000-126000 \mathrm{~g} / \mathrm{mol}$ ) solutions were made at a $45 \mathrm{wt} \%$ concentration in DMF (Fisher, Fair Lawn, NJ) and required a flow rate of $0.26 \mathrm{~mL} / \mathrm{h}$. The solution concentrations were chosen based on previous experience with electrospinning these polymers. Solution viscosity had to be sufficient enough to allow chain entanglements but not too high as to inhibit transferring the solution from the vial into the syringe.

Characterization-The electrospun fibers were characterized via FESEM (Hitachi S-4700). Accelerating voltage, magnification, and working distance are noted on the bottom of each micrograph. The fibers were electrospun directly onto aluminum SEM mounts. The FESEM was coupled with EDX (Oxford Inca X-ray Microanalysis) for elemental analysis of electrospun fibers. Multiphoton microscopy, (Zeiss LSM 510 NLO) was used to observe the location of the heparin-bound dye. The sample was illuminated using a titanium: sapphire laser (Mira 900F, Coherent). A fluorescence excitation wavelength of $747 \mathrm{~nm}$ and a KP685 emission filter were employed for these experiments. The $\mathrm{KP}$ filter is a short pass filter ( $\mathrm{KP}=$ short pass) that passes $\sim 325-685 \mathrm{~nm}$ wavelengths with $95 \%$ efficiency while blocking all relevant wavelengths above $685 \mathrm{~nm}$. Two types of objectives were used. An oil immersion type PlanNeofluar $40 \times / 1.3$ oil using differential interference contrast (DIC) was employed to obtain images in Figures 4, 5, 9, 10, and 12, and a Plan-Apochromat 63×/1.4 oil using phase contrast $(\mathrm{Ph} 3)$ was used to collect the image in Figure 7 . A $z$ series was made for each sample at 0.8 or $1.0 \mu \mathrm{m}$ sections. All images were processed using the system software (Zeiss LSM 5 Image Browser). Multiphoton images are represented in green to better illustrate the fluorescent properties of the fibers.

Toluidine Blue Assays-To determine the amount of heparin in the electrospun fibers, a colorimetric method was used. ${ }^{35}$ A toluidine blue solution was made by dissolving $25 \mathrm{mg}$ toluidine blue (Sigma Aldrich, St. Louis, MO) and $1 \mathrm{~g}$ of $\mathrm{NaCl}$ in $0.49 \mathrm{~mL}$ of $37 \% \mathrm{HCl}$ and $50 \mathrm{~mL}$ of water. Esterified sulfuric acid in heparin complexes with toluidine blue in a metachromatic reaction that is indicated by the presence of a blue color in the solution due to the complex. ${ }^{35}$ The toluidine blue that reacts with the heparin is removed from solution via extraction with hexanes $(5 \mathrm{~mL})$. The amount of unextracted toluidine blue remaining in the aqueous solution was determined by measuring the absorbance at $631 \mathrm{~nm}$ and, by difference, provides an estimate of the heparin concentration.

Samples were prepared by adding $1 \mathrm{mg}$ of electrospun fibers to a solution consisting of $0.8 \mathrm{~mL}$ of toluidine blue solution and $2.5 \mathrm{~mL}$ of $0.1 \% \mathrm{NaCl}$. The test tube was vortexed, and $5 \mathrm{~mL}$ of hexanes was added. The test tube was vortexed, and a phase separation between the hexanes 
(top layer) and toluidine blue solution (bottom layer) was apparent. After $10 \mathrm{~min}, 120 \mu \mathrm{L}$ of the toluidine blue solution was transferred into a cuvette, and absorbance measurements were taken. The control samples consisted of $1 \mathrm{mg}$ of either PEO or PLGA fibers, without heparin, to adjust for the amount of toluidine blue that may adhere to the fibers. The experiments were performed in duplicate.

Due to the difference in solubility of PEO versus PLGA in aqueous media, a note should be added that in the case of the heparin-containing PEO fibers, the toluidine blue assay is measuring the amount of heparin in the fibers and on the surface due to the fact that the PEO based fibers dissolve readily when placed in the toluidine blue solution. However, the PLGA based fibers do not dissolve over the time scale of the toluidine blue assay; therefore, the toluidine blue assay is most likely measuring the amount of heparin exposed on the surface of the fibers for the PLGA based samples.

Growth Factor Binding-Growth factor binding assays were employed to determine if the LMWH and PEG-LMWH electrospun samples were able to bind bFGF. All growth factor experiments were completed at room temperature in 24-well polystyrene assay plates (Corning Inc., Corning, NY), blocked with 3\% BSA (Sigma Aldrich, St. Louis, MO) in PBS (Invitrogen, Carlsbad, CA). All electrospun samples were washed with PBS at room temperature. Samples were placed in the wells and incubated with $500 \mu \mathrm{L}$ of a solution of basic fibroblast growth factor (recombinant, human bFGF, R\&D Systems, Minneapolis, MN, $10 \mathrm{ng} / \mathrm{mL}$ in PBS) for 2 h. After seven washes with $0.05 \mathrm{wt} \%$ Tween 20 in PBS (PBS-T), $500 \mu \mathrm{L}$ of a solution of bFGF antibody was added (R\&D Systems, Minneapolis, MN, $2 \mu \mathrm{g} / \mathrm{mL}$ in PBS) and incubated for 2 h. The samples were washed seven more times with PBS-T. The samples were then incubated for $30 \mathrm{~min}$ in a $500 \mu \mathrm{L}$ solution consisting of $1 \mu \mathrm{L}$ of horseradish peroxidase conjugated NeutrAvidin (Pierce, Rockford, IL, $1 \mathrm{mg} / \mathrm{mL}$ ), $8 \mathrm{~mL}$ of PBS, and $2 \mathrm{~mL}$ of SuperBlock Blocking Buffer (Pierce, Rockford, IL). The fibers were then washed seven times with PBS-T and incubated in $500 \mu \mathrm{L}$ of TMB substrate solution (Pierce, Rockford, IL) for 10-12 min. The absorbance, measured at $450 \mathrm{~nm}$, from the ELISA assay corresponds to the amount of growth factor bound to the electrospun fibers. All absorbance values were normalized with respect to a well treated with bFGF and antibody, as explained previously, but without any electrospun fibers. The experiments were performed in triplicate, with single measurements of three separately prepared samples.

\section{Results}

\section{Electrospinning Physical Mixtures of LMWH and PEO}

Initially, a physical mixture of PEO and LMWH was electrospun as a model to investigate if LMWH could be physically mixed into solution before electrospinning for successful incorporation into electrospun fibers. A 1:4 LMWH to PEO mixture (25 mg LMWH and 100 mg PEO) was dissolved in water and electrospun. The fibers (Figure 1a) resembled typical electrospun PEO fibers with an approximate diameter of 100-400 $\mathrm{nm}$ and were slightly beaded. The presence of microspheres with an average diameter of $1-5 \mu \mathrm{m}$ was also observed (Figure 1b). Two control solutions were made. One control consisted of a $10 \mathrm{wt} \% \mathrm{PEO} / \mathrm{H}_{2} \mathrm{O}$ and the other a $10 \mathrm{wt} \% \mathrm{LMWH} / \mathrm{H}_{2} \mathrm{O}$. The $10 \mathrm{wt} \% \mathrm{PEO} / \mathrm{H}_{2} \mathrm{O}$ solution electrospun to form PEO fibers with a diameter of 100-400 nm (Figure 2). The fibers were circular in their shape and contained beads (200-600 nm in diameter) but no microspheres were observed. The $10 \mathrm{wt} \% \mathrm{LMWH} /$ $\mathrm{H}_{2} \mathrm{O}$ control did not form fibers or microspheres, which is almost certainly due to the lack of entanglements in the negatively charged LMWH $(3000 \mathrm{~g} / \mathrm{mol})$. Attempts to electrospin higher MW heparin (12000 g/mol) also failed for similar reasons.

Toluidine blue assays were performed on the electrospun physical mixtures of PEO and LMWH to determine if LMWH was present in the sample. It was found that $86 \mu \mathrm{g}( \pm 7.15)$ of 
LMWH was present per mg of electrospun fibers. The presence of LMWH indicated by the toluidine blue assay is consistent with the presence of LMWH in the matrix, but does not indicate the uniformity of the glycosaminoglycan incorporation.

EDX was employed to determine the location of the heparin in the fiber matrix via detection of sulfur on the sulfated heparin; the results of the EDX analysis are outlined in Table 1. The presence of aluminum in the sample is due to the aluminum SEM mount used to hold the fibers. Multiple areas on the sample were tested, and the average measurements and standard deviation are displayed in Table 1 (at least three measurements were done on each sample to determine the average). PEO fibers served as a control for this experiment; EDX detected $46 \% \mathrm{C}, 5 \% \mathrm{O}$, and $49 \% \mathrm{Al}$ in the PEO fibers, consistent with the composition of PEO. The significant amount of $\mathrm{C}$ detected was most likely to due to carbon adhesive tab that was used to secure the fibers for imaging. Because LMWH could not be electrospun into a mat, a thin film was cast of 10 wt $\% \mathrm{LMWH} / \mathrm{H}_{2} \mathrm{O}$ and used as the second control The atomic composition detected via EDX was $23 \% \mathrm{C}, 40 \% \mathrm{O}, 17 \% \mathrm{~S}$, and $18 \% \mathrm{Na}$, consistent with the presence of LMWH in the sample (sodium is the counterion for the LMWH used in these studies). The 1:4 LMWH:PEO electrospun sample was tested in three different areas: the fiber portion, bead portion, and the microsphere. The fibers contained $42 \% \mathrm{C}, 5 \% \mathrm{O}$, and $59 \% \mathrm{Al}$. Since no sulfur or sodium was detected, the results suggest that either the fiber section of the matrix consists of primarily PEO or that the concentration of sulfur is too low for detection via EDX. Generally, the lower limit of detection for EDX is approximately $1 \%,{ }^{36}$ so it is possible that the LMWH may be incorporated into the fibers but at a concentration much lower than $1 \%$. The beaded section of the matrix was found to contain $61 \% \mathrm{C}, 13 \% \mathrm{O}, 26 \% \mathrm{Al}$, and $0.3 \% \mathrm{~S}$. The detection of the sulfur indicates that LMWH is present, at low concentrations, in the beaded areas of the electrospun mat. The microspheres were shown to contain $73 \% \mathrm{C}, 20 \% \mathrm{O}, 4 \% \mathrm{Al}, 1.2 \% \mathrm{~S}$, and $1.4 \% \mathrm{Na}$. The presence of sulfur and sodium imply that LMWH is present in the microspheres at higher concentrations than found in the beaded sections of the mat. The EDX results are consistent with the expected composition of the fibers, and the varying amount of sulfur in the different regions suggests that LMWH is not uniformly incorporated into the fibers via simple mixing.

\section{Electrospinning Dye-Labeled LMWH with PEO}

To visually map the location of the LMWH throughout the electrospun mat, a fluorescent dye, Alexa Fluor 350, was covalently attached to LMWH, and the fibers were analyzed via multiphoton microscopy; observation of fluorescence permits direct observation of the presence and location of LMWH throughout the electrospun matrix. PEO (10 wt \%) was mixed with Alexa Fluor 350-labeled LMWH (0.2 wt \%). The solution was electrospun and imaged via FESEM to determine if fiber formation was successful. Figure 3 a shows fibers with diameters of approximately 100-200 nm. The fibers were slightly beaded with the presence of a few very large beads approximately $6 \mu \mathrm{m}$ in diameter (Figure $3 \mathrm{~b}$ ).

The fibers were then analyzed via multiphoton microscopy to determine if the LMWH-dye molecules were incorporated into the matrix and to determine their distribution in the matrix. Electrospun PEO fibers were used as a control; results for the control are shown in Figure 4. Figure 4a shows data in reflection mode and confirms that fibers were present on the glass coverslip. Figure $4 \mathrm{~b}$ shows two small areas of fluorescence that are likely impurities in the PEO or dust in the sample, but confirms the complete lack of background fluorescence in the PEO fibers.

Figure 5 presents multiphoton microscopy data for electrospun fibers of the LMWH-dye/PEO mixture. The results demonstrate the fluorescence of the fibers, indicating the presence of LMWH. The dark regions in the image, as in all of the multiphoton images, are areas that are out of focus due to the uneven contour of the electrospun sample. In contrast to SEM and EDX 
results, the multiphoton results also suggest that the LMWH-dye is present not only in the beaded sections of the matrix but also in the fiber regions. Figure 5 shows the fluorescence of the fibers due to LMWH-dye incorporation. Z-stack images were also taken (not shown) to ensure that the LMWH-dye is present throughout the entire depth of the matrix and is not localized in any one area. Since the nanometer fiber diameters approach the limit of resolution on the multiphoton microscope, the exact location of the heparin within each individual fiber is unknown. However, all multiphoton microscopy images indicate that LMWH is present throughout the depths of the electrospun matrix, suggesting a fairly uniform dispersion of LMWH. Measurement of LMWH via toluidine blue assay confirmed the presence of approximately $4.3 \mu \mathrm{g}( \pm 0.923)$ of LMWH per mg of electrospun sample. A direct comparison cannot be made between the amount of LMWH in this sample and the amount in the physical mixtures of LMWH and PEO due to differing amounts of LMWH in the initial solutions. The physical mixtures contained $25 \mathrm{wt} \% \mathrm{LMWH}$, whereas this sample contained only $0.2 \mathrm{wt} \%$ due to sample availability. Nevertheless, these results suggest the opportunities for facile incorporation of bioactive polysaccharides into electrospun matrices.

\section{Incorporation of PEG-LMWH-Dye in PEO for Electrospinning}

Based on the above results, we also tested the incorporation of a PEG-LMWH conjugate into electrospun fibers via similar methods. In particular, we were interested in improving the uniformity of heparin in the fibrous matrix and in determining strategies that would increase the compatibility of heparin with the PEO and perhaps slow its release. To address these issues, LMWH was covalently attached to the termini of a 4-arm star PEG and evaluated for its incorporation into an electrospun matrix. PEG-LMWH-dye $(0.2 \mathrm{wt} \%)$ was mixed with PEO $(10 \mathrm{wt} \%)$ in water. The electrospun fibers from this solution were analyzed via FESEM, with results shown in Figure 6. As is shown in Figure 6, parts a and b, the resulting fibers were 100$300 \mathrm{~nm}$ in diameter with the presence of microspheres $(1-3 \mu \mathrm{m})$. The morphology of the PEGLMWH fibers was very similar to those produced from the earlier samples with free LMWH. In both electrospun mats, fibers are in the nanometer size range, and the onset of bead formation is observed by the roughness of the fibers (Figures $3 a$ and $6 a$ ). Both samples also contain large beads or microspheres in the micron size range that are randomly distributed throughout the electrospun membrane.

The fiber matrix was then analyzed via multiphoton microscopy to confirm that the PEGLMWH-dye molecule was incorporated in the electrospun fibers; representative results are shown in Figure 7. Similar to the results in Figure 5, the data shown here also indicate that the fibers are evenly fluorescent, showing that the PEG-LMWH was evenly distributed in the PEO fiber matrix. Again, the large nonfluorescent areas result from the uneven contour of the electrospun fibrous mat. These fibers were found, via toluidine blue assay, to contain $3.5 \mu \mathrm{g}$ $( \pm 0.397)$ of heparin per $\mathrm{mg}$ of electrospun fibers. These results may suggest a slight improvement in LMWH incorporation in comparison to the LMWH/PEO fibers and may suggest that PEG-LMWH permits the incorporation of slightly more heparin than in the fibers produced using free heparin.

These studies confirm that mixtures of polymers and polysaccharides (PEG-LMWH and PEO) can be electrospun from a solution to produce fibers containing both compounds evenly distributed throughout the matrix. The increased number of beads and microspheres present in the heparin-containing PEO fibers may result from a reduction in polymer entanglement due to the presence of the PEG-LMWH in the sample; an increase in beaded fibers as a result of decreased polymer entanglement has been previously reported, ${ }^{37}$ and additional reports have shown a direct relationship between viscosity and fiber formation. ${ }^{38-40}$ Lack of sufficient polymer chain entanglements can inhibit fiber formation completely or can result in freestanding beads or spheres. ${ }^{41}$ 


\section{Changing the Carrier Polymer}

Although PEO proved to be useful for the incorporation of LMWH into electrospun fibers, it was of interest to determine the ability of incorporating LMWH into multiple types of fibers via these simple protocols. PLGA was chosen as an alternate carrier polymer for the LMWH systems due to the success of PLGA in biomaterial applications. ${ }^{24-26}$ PLGA/DMF (45 wt \%) was electrospun with LMWH-dye $(0.2 \mathrm{wt} \%)$ and also with PEG-LMWH-dye $(0.2 \mathrm{wt} \%)$. Due to differences in molecular weight and viscosity, the concentration of carrier polymer is different than those used for PEO electrospinning, but all other processing conditions remained the same. The electrospun PLGA fibers are approximately 400-800 $\mathrm{nm}$ in diameter (Figure 8a). SEM revealed that fiber morphology was not affected by the incorporation of LMWHdye or PEG-LMWH-dye (Figure 8, parts b and c).

These fibers were also characterized via multiphoton microscopy to confirm that changing the carrier polymer did not affect the incorporation or distribution of heparin throughout the electrospun matrix. The PLGA electrospun mat, shown in Figure 9a under transmitted light, does not fluoresce to any significant extent. As encountered previously with PEO fibers, there is a small area of fluorescence in Figure 9b, which is likely due to polymer impurities or dust on the sample. The images shown in Figure 10 reveal that both LMWH-dye and PEG-LMWHdye were incorporated into the PLGA matrix, and z-stack multiphoton images confirm that the heparin is present throughout the depths of the matrices.

To quantitatively determine the amount of LMWH present, toluidine blue assays were also conducted on the fiber matrices. The toluidine blue assay indicated that there is approximately $8.41 \mu \mathrm{g}( \pm 0.532)$ of LMWH present per mg of electrospun LMWH/PLGA sample. The PEGLMWH/PLGA sample contained $9.50 \mu \mathrm{g}( \pm 0.382)$ of LMWH per mg of electrospun fibers. Thus, both LMWH and PEG-LMWH-containing scaffolds contain similar amounts of heparin after electrospinning. The results of the FESEM, multiphoton microscopy, and the toluidine blue assays suggest that the carrier polymer can be changed without affecting the incorporation of heparin or fiber morphology.

Since bFGF is known to bind heparin, we conducted an investigation to determine if there are any functional differences in growth factor binding between LMWH/PLGA fibers versus PEGLMWH/PLGA fibers. An ELISA format was employed to characterize the binding of bFGF in a series of samples: a blank well, PLGA fibers alone, LMWH/PLGA fibers, and PEGLMWH/PLGA fibers. The optical density measurements, at a wavelength of $450 \mathrm{~nm}$, resulted in the following absorbance values relative to the control (increasing absorbance values correlate with increasing growth factor binding): PLGA fibers $1.59( \pm 0.055), \mathrm{LMWH} / \mathrm{PLGA}$ fibers $2.46( \pm 0.146)$, and PEG-LMWH/PLGA fibers $3.59( \pm 0.106)$. Figure 11 shows that there are significant differences in bFGF binding between the LMWH/PLGA and PEG-LMWH/ PLGA fibers, despite the fact that the toluidine blue assays show that the two matrices contain similar amounts of LMWH after electrospinning. The matrices are washed multiple times during the binding assays, which may result in a significant loss of LMWH via diffusion. Since both the LMWH and PEG-LMWH samples began with similar amounts of heparin but did not exhibit the same level of bFGF binding, these data suggest that the PEG-LMWH may be retained in the electrospun fibers more effectively than LMWH alone. FESEM characterization of the electrospun membranes after the growth factor binding assay revealed that the PLGA fibers exhibited no visible signs of degradation during the time frame of the growth factor binding study (data not shown), which corroborates that differences in growth factor binding likely result from differential LMWH retention rather than from variations in fiber degradation between samples. Characterization of the fibers after completion of the growth factor binding assays was conducted via multiphoton microscopy. Figure 12 shows the LMWH retention within the electrospun fibers after the growth factor assay plus an additional $24 \mathrm{~h}$ of incubation in PBS. Slight differences in the amount of LMWH within the PLGA/LMWH versus the 
PLGA/PEG-LMWH fibers directly after the growth factor assay were observed via multiphoton microscopy; these differences were much more pronounced with an additional 24 $\mathrm{h}$ incubation in PBS. The differences in the amount of LMWH retained in the fibers were also observed throughout the depth of the matrix, as confirmed via z-stack images (data not shown). Detailed release profiles for the LMWH and PEG-LMWH are currently under investigation.

\section{Discussion}

These results indicate that LMWH can be successfully incorporated into electrospun fibers for possible biomedical applications. Other research groups ${ }^{3,42}$ have demonstrated the ability to incorporate therapeutic molecules into biocompatible electrospun fibers for use in drug delivery applications. For example, tetracycline has been incorporated in a solution of poly (lactic acid) (PLLA) and poly(ethylene-co-vinyl acetate) and electrospun in a fiber membrane to study the release profile of the fiber-encapsulated drug. ${ }^{3}$ PLLA has also been studied as a matrix to incorporate the drugs rifampin (to treat tuberculosis) and paclitaxel (anti-cancer drug). ${ }^{42}$ Polymers loaded with both of these therapeutic molecules could be fabricated into nanometer to micron diameter electrospun PLLA fibers and used for delivery of the drug. ${ }^{42}$

A limited number of studies have also been done on the preprocessing functionalization of electrospun fibers to confer a specific property to the electrospun mat. Chaikof and coworkers, for example, have varied the degree of acrylate functionalization of an elastin-mimetic protein polymer in order facilitate cross-linking of the electrospun elastin-mimetic fibers for possible use in tissue engineering scaffolds. ${ }^{43,44}$ Kenway et al. modified poly(vinyl phenol) by introducing sulfonic groups on the phenyl ring of the polymer in order to produce an electrospun mat with a high degree of antimicrobial activity. ${ }^{4}$ Jiang and co-workers synthesized poly (ethylene glycol)- $g$-chitosan (PEG- $g$-CHN) and mixed it with a polymer solution of poly (lactide- $c o$-glycolide) to electrospin fibers. ${ }^{14}$ The PEG- $g$-CHN was covalently conjugated to ibuprofen before electrospinning, and it was determined that the covalent conjugation of ibuprofen to PEG- $g$-CHN resulted in a slow release of the drug over a 16 day period. Although these previous studies demonstrate the utility of functionalizing electrospun fibers, the functionalization of electrospun fibers to confer specific biological activity would be particularly advantageous for many biomaterial applications, as such modification would permit the fabrication of biomaterials that are structurally relevant and that possess properties designed to treat or address specific problems. In particular, the development of simple methods to produce multifunctional electrospun fibers would advance the development of electrospun fibers for biomedical applications. However, the fabrication of biologically active fibers through functionalization strategies is a fairly new and unexplored area in the field of electrospinning, and to the best of our knowledge, electrospun fibers have not been investigated for their ability to incorporate growth factor binding glycosaminoglycans, such as heparin, in a manner designed to control release.

The incorporation of heparin in the electrospun matrix is of functional importance owing to heparin's role as a binding site for many growth factors. The binding of growth factors to heparin serves to help preserve and store growth factors until release and may sustain their bioactivity. ${ }^{30,45-48}$ Although LMWH was indicated to be incorporated into fibers with simple mixing in our investigation, the conjugation of LMWH to the star PEG slightly improved the amount of heparin incorporated into the fiber matrix and permitted a significantly improved amount of growth factor binding to these matrices, which was suggested to result from an improved retention of heparin in the fibers. If the use of such PEG-LMWH conjugates also slows the release of heparin over extended periods of time, it will allow for a slower, more sustained release of growth factor, which is often required to achieve desired biological outcomes. For example, Sakiyama-Elbert et al. showed that the binding of heparin to bFGF in a fibrin matrix provided a slower release of growth factor over a duration of 6 weeks to facilitate 
nerve regeneration. ${ }^{30}$ Schroeder-Tefft et al. demonstrated that TGF- $\beta 2$ can be stabilized and its biologically activity preserved when it is bound to heparin in a collagen matrix. ${ }^{17}$

The conjugation of heparin to a short polymeric chain therefore permits facile functionalization of fibers and also allows for retention and significantly slowed release of heparin from the matrix over time. Given the importance of co-presentation of the heparin with growth factors at the growth factor receptor, ${ }^{34}$ these systems also offer the potential advantage of improving growth factor activity over systems in which heparin is covalently attached directly to the matrix and can therefore not be co-released with growth factor. The rate of release of heparin (and therefore growth factors) may also be modified via modifications in the polymer conjugate's molecular weight over a wide range, and coupled with the greater entanglement likely for flexible polymer chains, this may offer advantages over simple incorporation of a higher molecular weight heparin in fibers. In addition, the controlled incorporation and delivery of other and potentially smaller bioactive molecules, such as peptides, would also be widely possible via the incorporation of other similar PEG bioconjugates into these fibers. The ability to simply mix various PEG conjugates in the electrospinning solution also offers advantages for creating multifunctional fibers.

The PEG-LMWH molecules used in these electrospinning studies have previously been demonstrated to be capable of sequestering growth factors in hydrogels and delivering them in bioactive form to increase the proliferation rate of human umbilical vein endothelial cells (unpublished results). The amount of LMWH in these electrospun fiber matrices $(3-86 \mu \mathrm{g})$ is also similar to that in hydrogels produced with these polymers; the hydrogels can effectively release bFGF for time periods of at least 16 days. ${ }^{34}$ These results are therefore significantly promising for the potential of delivering similarly active growth factors from electrospun matrices.

\section{Conclusions}

The results of this investigation reveal that LMWH functionalized PEG molecules can be easily electrospun into fibers. These fibers have nanometer diameters when electrospun using either PEO or PLGA as carrier polymers, and multiphoton microscopy confirmed the presence of LMWH throughout the depth of the electrospun matrix. The amount of LMWH per mg of fibers was determined to range from 3.5 to $85 \mu \mathrm{g}$ depending on the sample examined. Although both LMWH and PEG-LMWH can be processed into fibers, the incorporation of PEG-LMWH resulted in functional advantages such as improved growth factor binding, which likely results from improvements in retention of the PEG-LMWH in the fibers over the LMWH alone. The PEG-LMWH is retained in the fibers for at least 14 days, in contrast to LMWH, which is almost completely released from the fibers after $24 \mathrm{~h}$. A slower release of LMWH, and any coupled growth factors, would allow for drug delivery over a period of time that would more closely match the time scales needed for tissue repair or regeneration if the fibers are used in biomedical applications. The use of electrospinning as the fabrication method for this biologically functionalized matrix proved useful owing to its ability to allow for the incorporation of LMWH, ease of fabrication, and use of small amounts of material. The ability to scale electrospinning from the laboratory to the industrial scale offers opportunities to produce matrices for biomaterials applications. The electrospun matrix also meets many requirements in its porosity and 3-D nature to make the fibers suitable for drug targeting and tissue engineering applications. Such simple strategies for creating electrospun matrices that are both structurally relevant and biologically active could find increased utility in a variety of biomedical applications. The effect of these matrices on cellular behavior is currently under investigation. 


\section{Acknowledgments}

The authors thank Dr. Kirk Czymmek and Debbie Powell for their assistance with multiphoton microscopy and FESEM. We also thank NSF-NIRT (DMR-0210223), NASA (NAG8-01923), and NIH (1R01EB003172-01) for financial support. In addition, C.L.C. thanks the University of Delaware IGERT program for partial support during this project.

\section{References and Notes}

1. Boland ED, Wnek GE, Simpson DG, Pawlowski KJ, Bowlin GL. J Macromol Sci-Pure Appl Chem 2001;38:1231-1243.

2. Boland ED, Bowlin GL, Simpson DG, Wnek GE. Abstr Papers Am Chem Soc 2001;222:31-PMSE.

3. Kenawy ER, Bowlin GL, Mansfield K, Layman J, Simpson DG, Sanders EH, Wnek GE. J Controlled Release 2002;81:57-64.

4. Kenawy ER, Abdel-Fattah YR. Macromol Biosci 2002;2:261-266.

5. Li WJ, Laurencin CT, Caterson EJ, Tuan RS, Ko FK. J Biomed Mater Res 2002;60:613-621. [PubMed: 11948520]

6. Luu YK, Kim K, Hsiao BS, Chu B, Hadjiargyrou M. J Controlled Release 2003;89:341-353.

7. Matthews JA, Wnek GE, Simpson DG, Bowlin GL. Biomacromolecules 2002;3:232-238. [PubMed: 11888306]

8. Wnek GE, Carr ME, Simpson DG, Bowlin GL. Nano Lett 2003;3:213-216.

9. Xu CY, Inai R, Kotaki M, Ramakrishna S. Biomaterials 2004;25:877-886. [PubMed: 14609676]

10. Yoshimoto H, Shin YM, Terai H, Vacanti JP. Biomaterials 2003;24:2077-2082. [PubMed: 12628828]

11. Zong XH, Kim K, Fang DF, Ran SF, Hsiao BS, Chu B. Polymer 2002;43:4403-4412.

12. www.eSpintechnologies.com, 2005.

13. www.donaldson.com, 2005.

14. Jiang HL, Fang DF, Hsiao BJ, Chu BJ, Chen WL. J Biomater Sci-Polym Ed 2004;15:279-296. [PubMed: 15147162]

15. Bhattarai SR, Bhattarai N, Yi HK, Hwang PH, Cha DI, Kim HY. Biomaterials 2004;25:2595-2602. [PubMed: 14751745]

16. Sakiyama-Elbert SE, Hubbell JA. J Controlled Release 2000;65:389-402.

17. SchroederTefft JA, Bentz H, Estridge TD. J Controlled Release 1997;48:29-33.

18. Ishihara M, Obara K, Ishizuka T, Fujita M, Sato M, Masuoka K, Saito Y, Yura H, Matsui T, Hattori H, Kikuchi M, Kurita A. J Biomed Mater Res Part A 2003;64A:551-559.

19. Zisch AH, Lutolf MP, Hubbell JA. Cardiovasc Pathol 2003;12:295-310. [PubMed: 14630296]

20. Griffith LG. Acta Mater 2000;48:263-277.

21. Irvine DJ, Mayes AM, Satija SK, Barker JG, Sofia-Allgor SJ, Griffith LG. J Biomed Mater Res 1998;40:498-509. [PubMed: 9570083]

22. Li J, Ni XP, Leong KW. J Biomed Mater Res Part A 2003;65A:196-202.

23. Seal BL, Otero TC, Panitch A. Mater Sci Eng R-Rep 2001;34:147-230.

24. Hasirci V, Lewandrowski K, Gresser JD, Wise DL, Trantolo DJ. J Biotechnol 2001;86:135-150. [PubMed: 11245902]

25. Karakecili AG, Gumusderelioglu M. J Biomater Sci-Polym Ed 2002;13:185-196. [PubMed: 12022749]

26. Miller DC, Thapa A, Haberstroh KM, Webster TJ. Biomaterials 2004;25:53-61. [PubMed: 14580908]

27. Ishihara M, Sato M, Hattori H, Saito Y, Yura H, Ono K, Masuoka K, Kikuchi M, Fujikawa K, Kurita A. J Biomed Mater Res 2001;56:536-544. [PubMed: 11400131]

28. Guan R, Sun XL, Hou SJ, Wu PY, Chaikof EL. Bioconjugate Chem 2004;15:145-151.

29. Lieberman JR, Daluiski A, Einhorn TA. J Bone Joint Surg-Am Volume 2002;84A:1032-1044.

30. Sakiyama-Elbert SE, Hubbell JA. J Controlled Release 2000;69:149-158.

31. Seal BL, Panitch A. Biomacromolecules 2003;4:1572-1582. [PubMed: 14606882]

32. Hennink WE, van Nostrum CF. Adv Drug Delivery Rev 2002;54:13-36. 
33. Casper, CL.; Yamaguchi, N.; Chase, DB.; Rabolt, JF.; Kiick, KL. Functionalized electrospun fibers for potential use in biomedical applications. Vol. 91. Philadelphia, PA: 2004. p. 1030

34. Yamaguchi N, Kiick KL. Biomacromolecules. 2005accepted

35. Zhou D, Too CO, Wallace GG. React Funct Polym 1999;39:19-26.

36. Czymmek, K. Private communication, 2004.

37. Fong H, Chun I, Reneker DH. Polymer 1999;40:4585-4592.

38. Koski A, Yim K, Shivkumar S. Materials Lett 2004;58:493-497.

39. Son WK, Youk JH, Lee TS, Park WH. Polymer 2004;45:2959-2966.

40. Casper, CL.; Yang, W.; Farach-Carson, MC.; Rabolt, JF. ACS Symposium Series Book. 2004. submitted, Polymeric Nanofibers

41. Reyderman L, Stavchansky S. Int J Pharm 1995;124:75-85.

42. Jing Z, Xu XY, Chen XS, Liang QZ, Bian XC, Yang LX, Jing XB. J Controlled Release 2003;92:227231.

43. Huang L, McMillan RA, Apkarian RP, Pourdeyhimi B, Conticello VP, Chaikof EL. Macromolecules 2000;33:2989-2997.

44. Nagapudi K, Brinkman WT, Leisen JE, Huang L, McMillan RA, Apkarian RP, Conticello VP, Chaikof EL. Macromolecules 2002;35:1730-1737.

45. Roghani M, Mansukhani A, Dellera P, Bellosta P, Basilico C, Rifkin DB, Moscatelli D. J Biol Chem 1994;269:3976-3984. [PubMed: 8307953]

46. Chintala SK, Miller RR, McDevitt CA. Arch Biochem Biophys 1994;310:180-186. [PubMed: 8161203]

47. Mach H, Volkin DB, Burke CJ, Middaugh CR, Linhardt RJ, Fromm JR, Loganathan D, Mattsson L. Biochemistry 1993;32:5480-5489. [PubMed: 7684608]

48. Vlodavsky I, Fuks Z, Ishaimichaeli R, Bashkin P, Levi E, Korner G, Barshavit R, Klagsbrun M. J Cellular Biochem 1991;45:167-176. [PubMed: 1711529] 

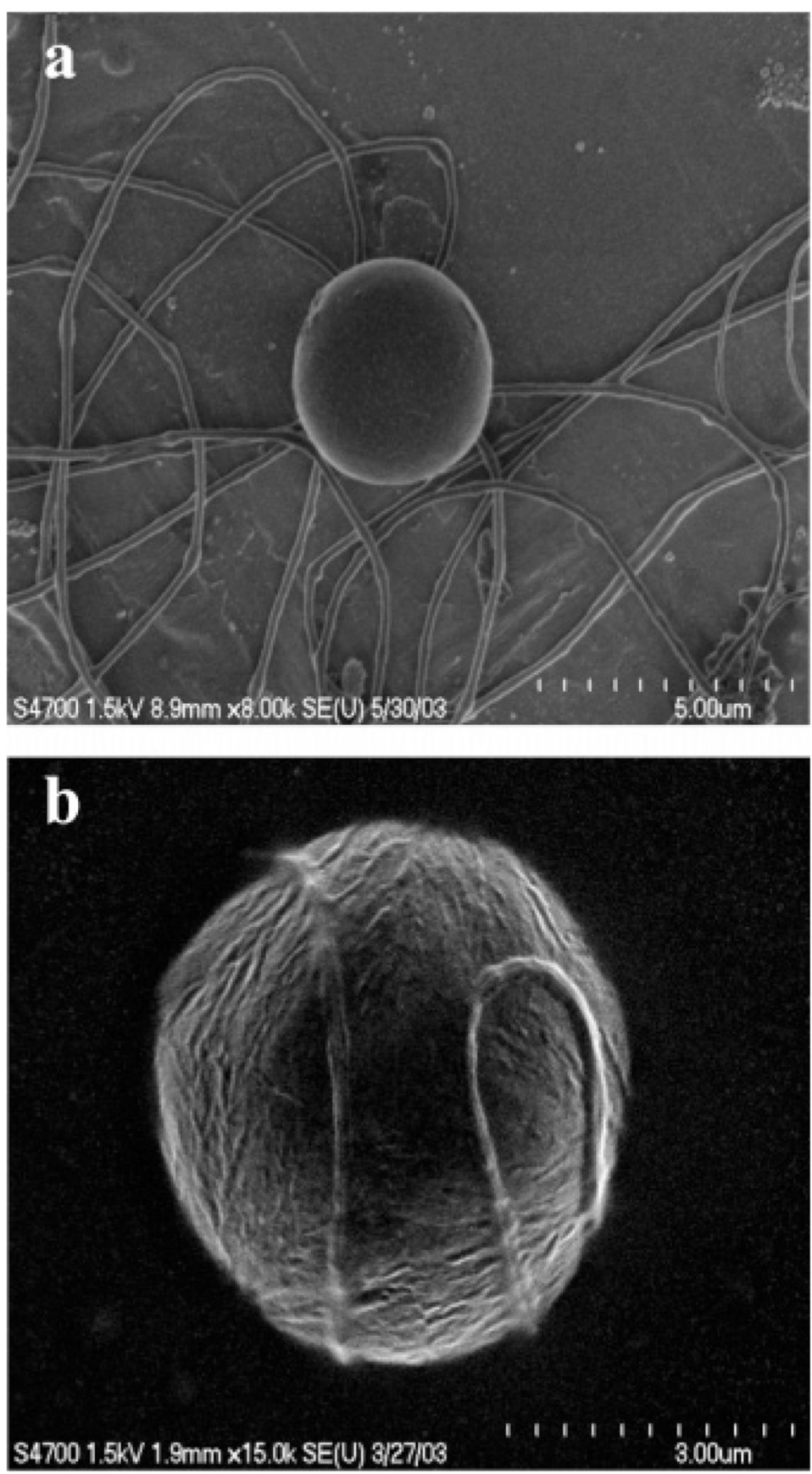

Figure 1.

Scanning electron micrographs of (a) Electrospun 1:4 LMWH:PEO and (b) Higher magnification of microsphere present on 1:4 LMWH:PEO electrospun sample. 


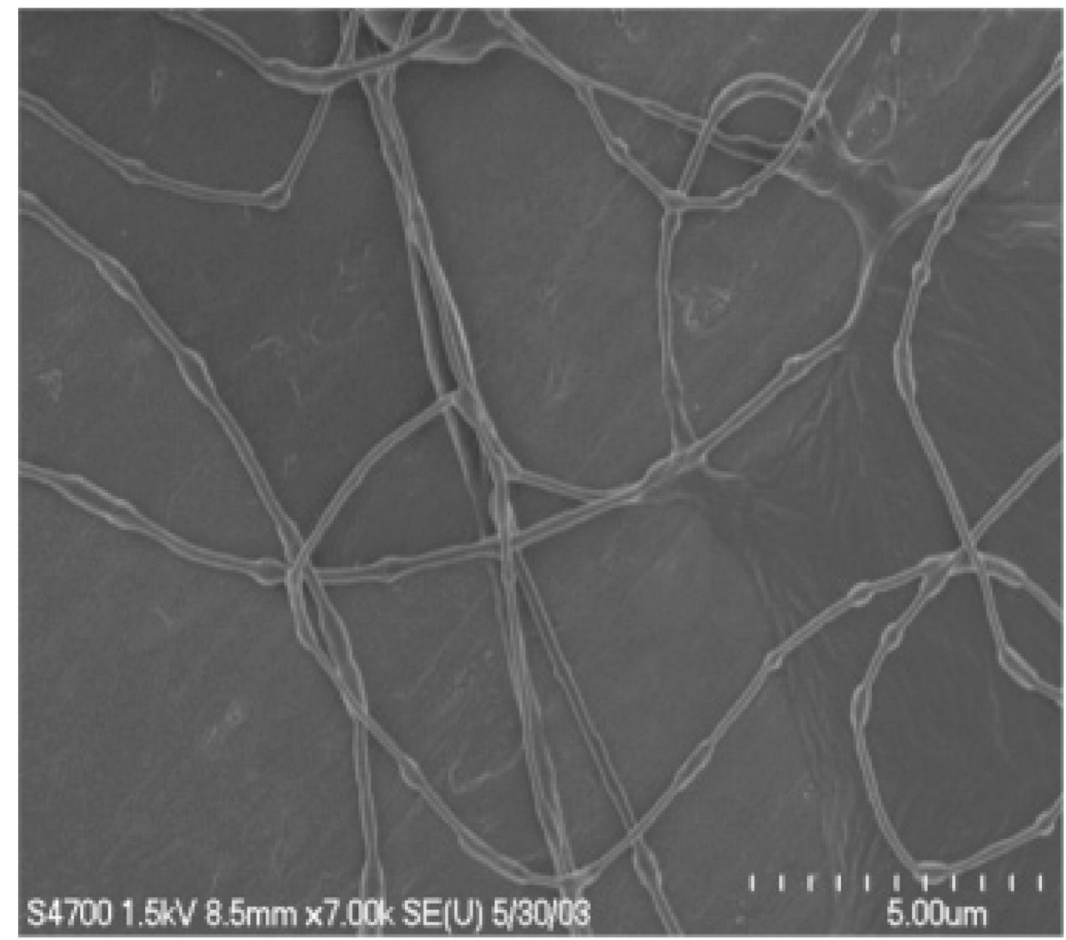

Figure 2.

Scanning electron micrograph of $10 \mathrm{wt} \% \mathrm{PEO} / \mathrm{H} 2 \mathrm{O}$ electrospun fibers (control). 

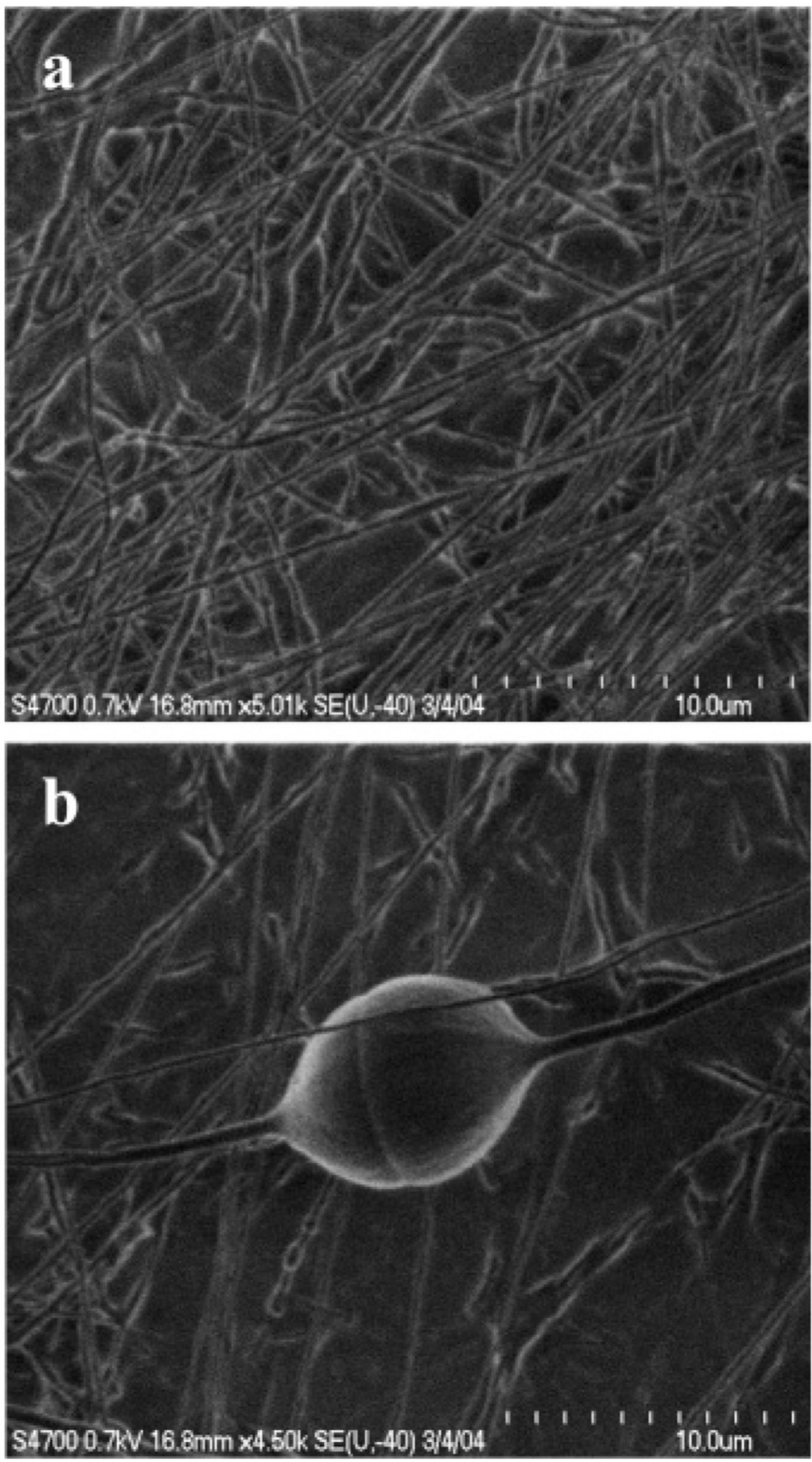

Figure 3.

Scanning electron micrographs of (a) PEO/LMWH-dye electrospun fibers and (b) beaded section of the fiber. 


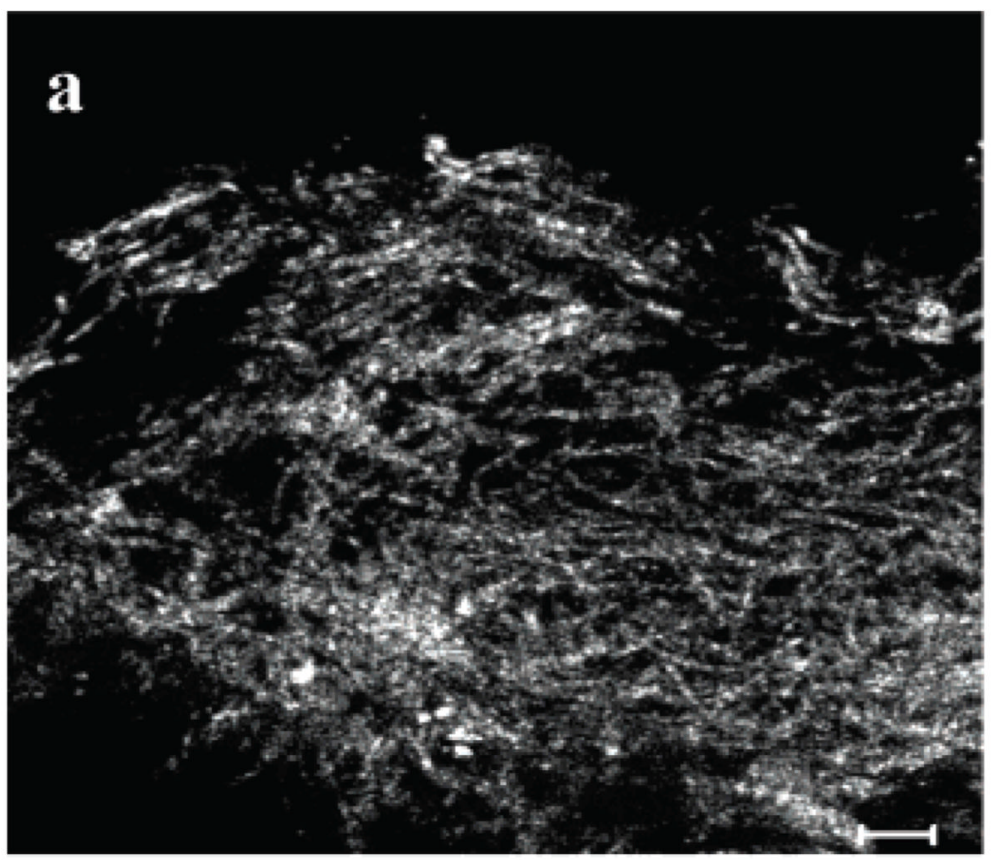

\section{b}

Figure 4.

Multiphoton microscopy images of electrospun PEO fibers (a) reflection mode and (b) fluorescence ( $5 \mu \mathrm{m}$ scale bars). 


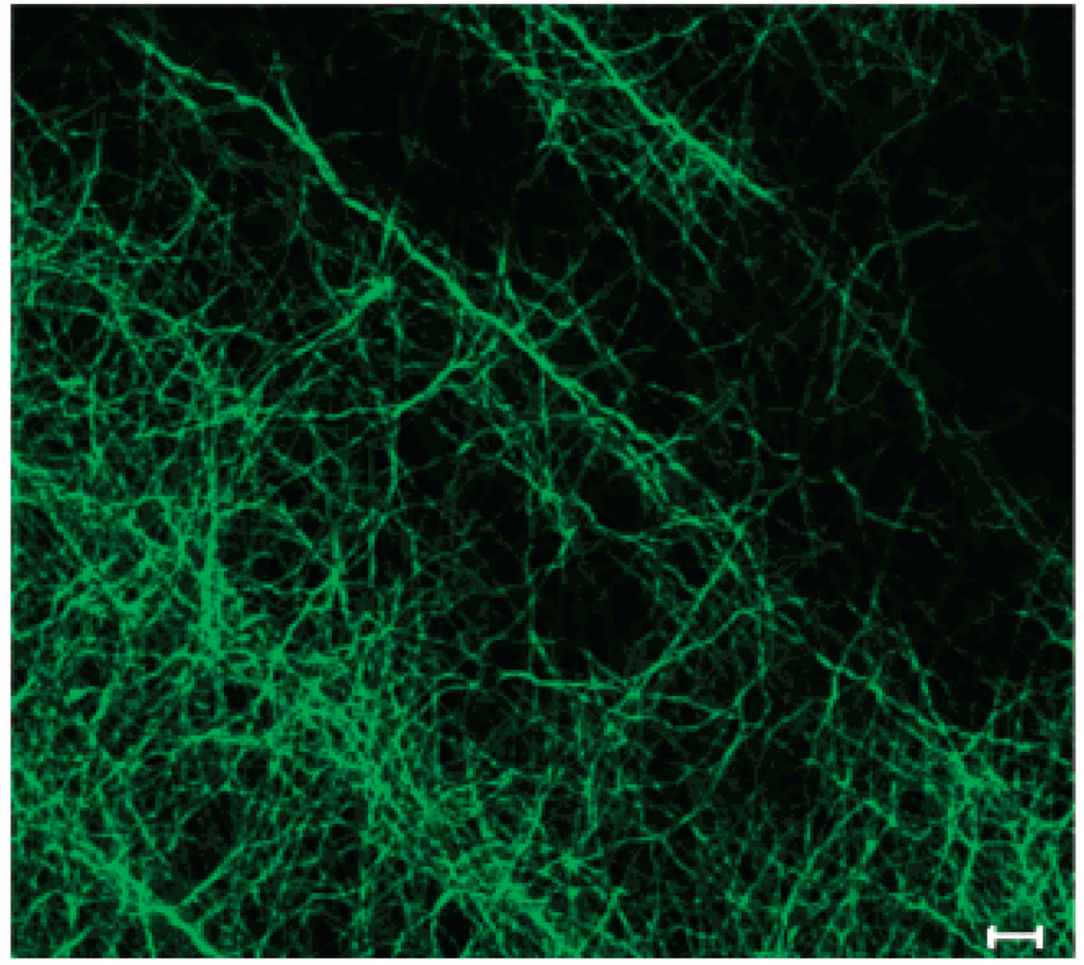

Figure 5.

Multiphoton microscopy image of electrospun PEO/LMWH-dye fibers ( $5 \mu \mathrm{m}$ scale bar). 

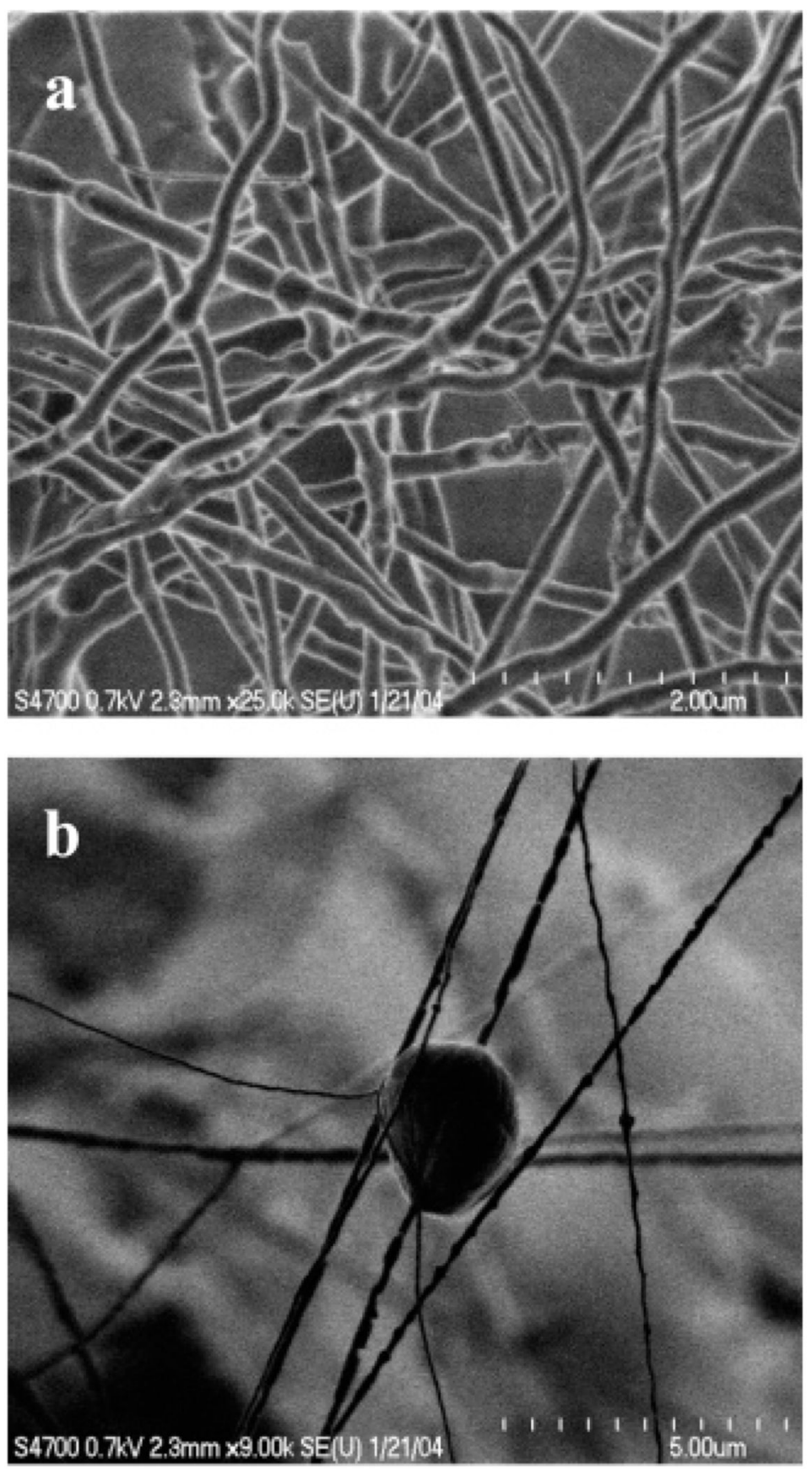

Figure 6.

Scanning electron micrographs of electrospun PEO/PEG-LMWH-dye (a) fibers and (b) beaded section of the fiber. 


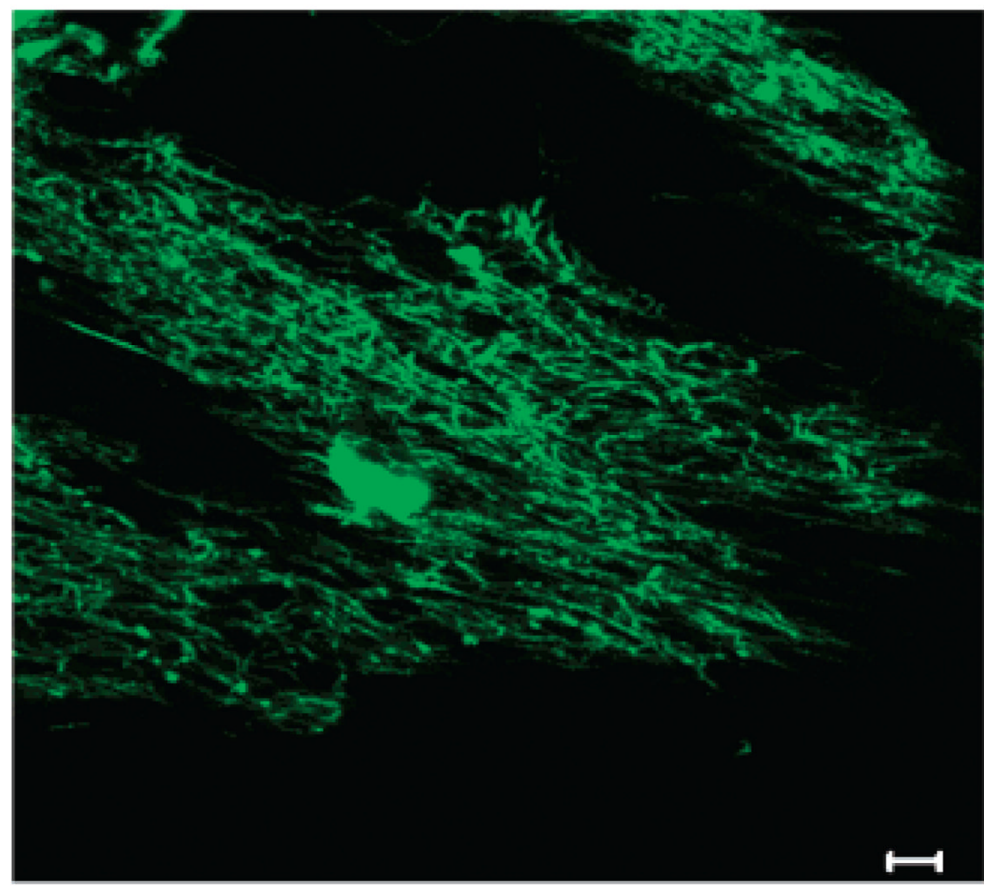

Figure 7.

Multiphoton microscopy image of PEG-LMWH-dye/PEO electrospun fibers (5 $\mu \mathrm{m}$ scale bar). 

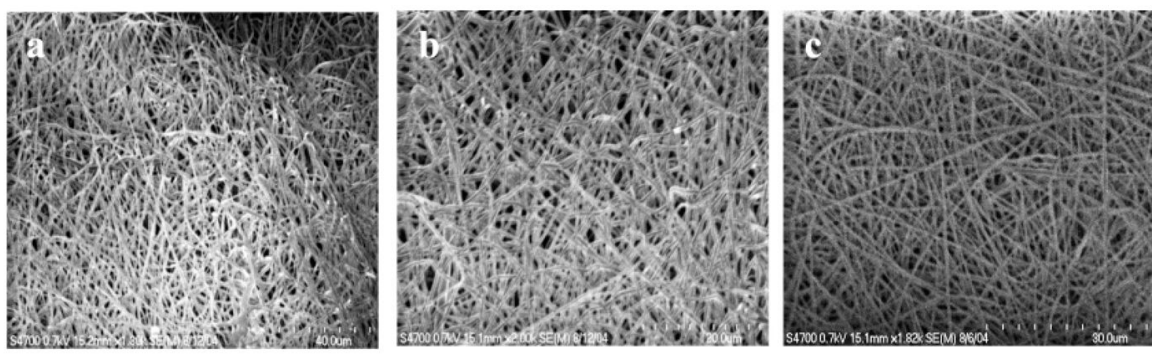

Figure 8.

Scanning electron micrographs of (a)PLGA electrospun fibers, (b) PLGA/LMWH-dye fibers, and (c) PLGA/PEG-LMWH-dye fibers. 

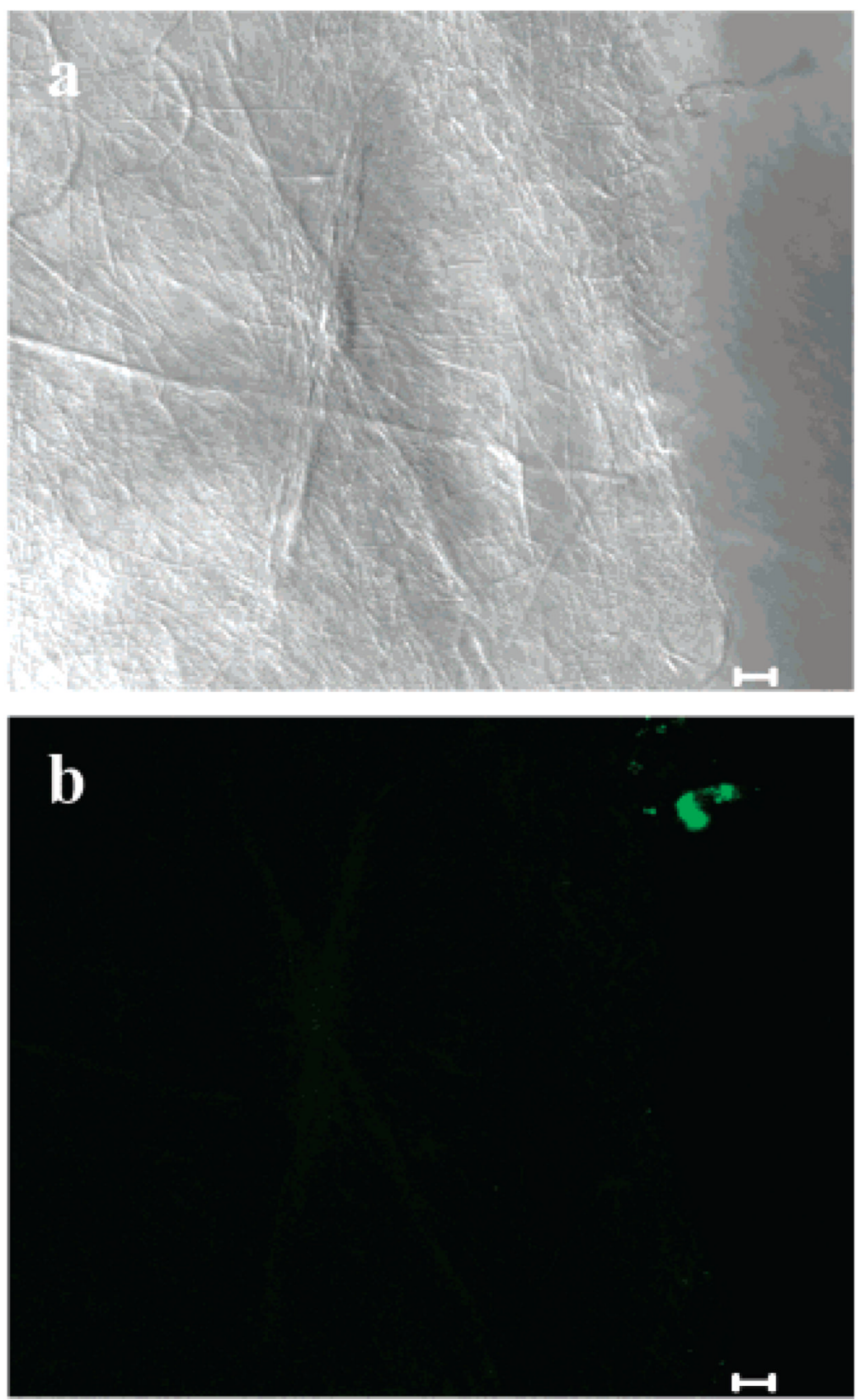

Figure 9.

Multiphoton microscopy images of electrospun PLGA fibers (a) transmitted light and (b) fluorescence $(10 \mu \mathrm{m}$ scale bars $)$. 

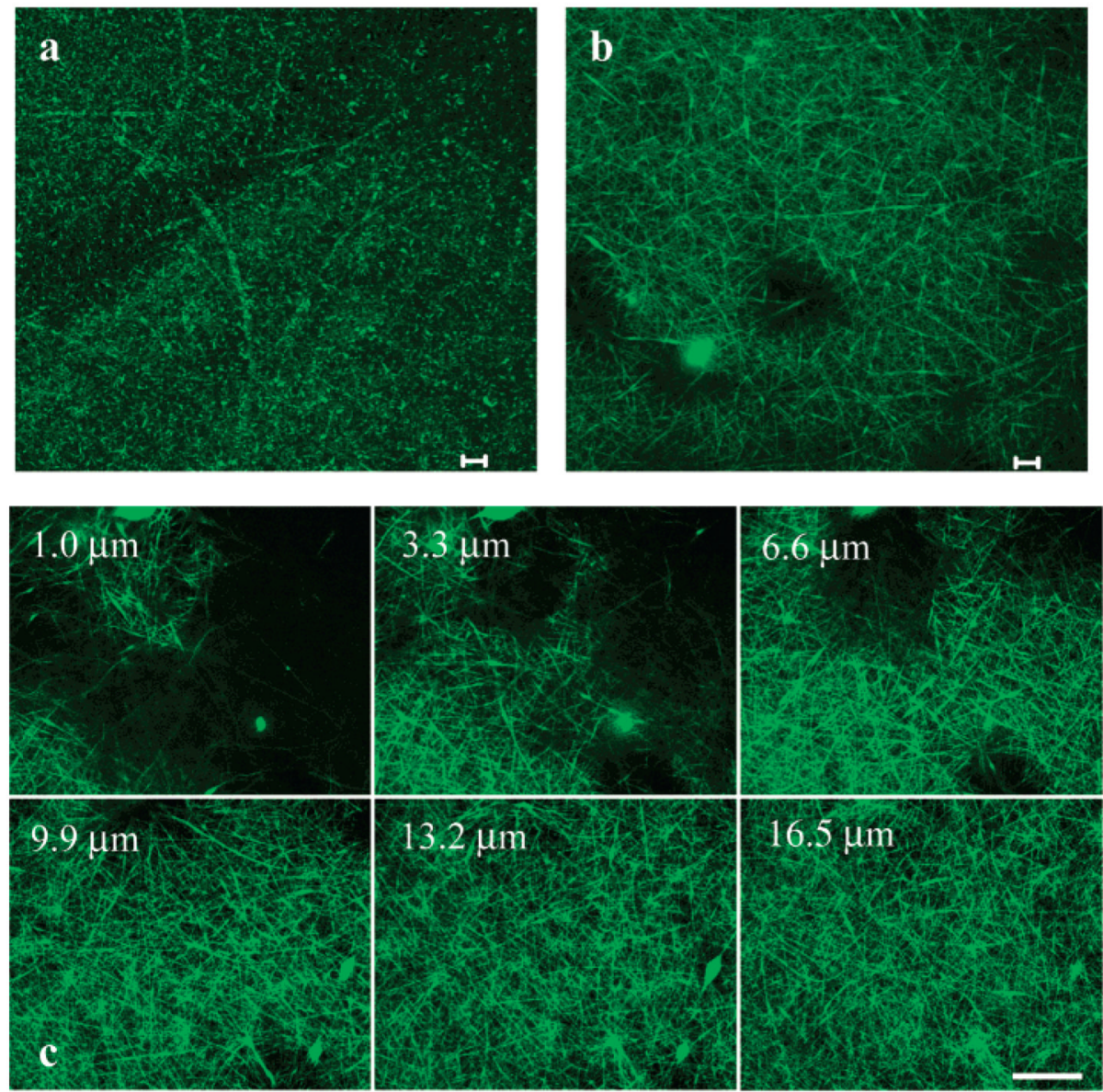

Figure 10.

Multiphoton microscopy images of electrospun (a) LMWH-dye/PLGA fibers (10 $\mu \mathrm{m}$ scale bar), (b) PEG-LMWH-dye/PGLA fibers (10 $\mu \mathrm{m}$ scale bar) and (c) z-stack of PEG-LMWH/ PLGA fibers $(20 \mu \mathrm{m}$ scale bar). 
bFGF Binding on Electrospun Matrices

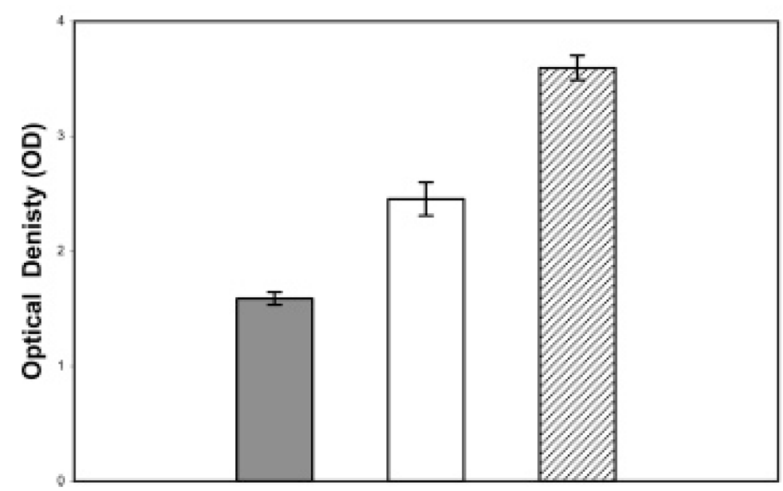

口PLGA Fibers

口LMWH/PLGA Fibers

囚PEG-LMWH/PLGA

Fibers

Figure 11.

bFGF binding on electrospun fibers. Each bar indicates \pm standard deviation of the average of three measurements. 


\section{a}
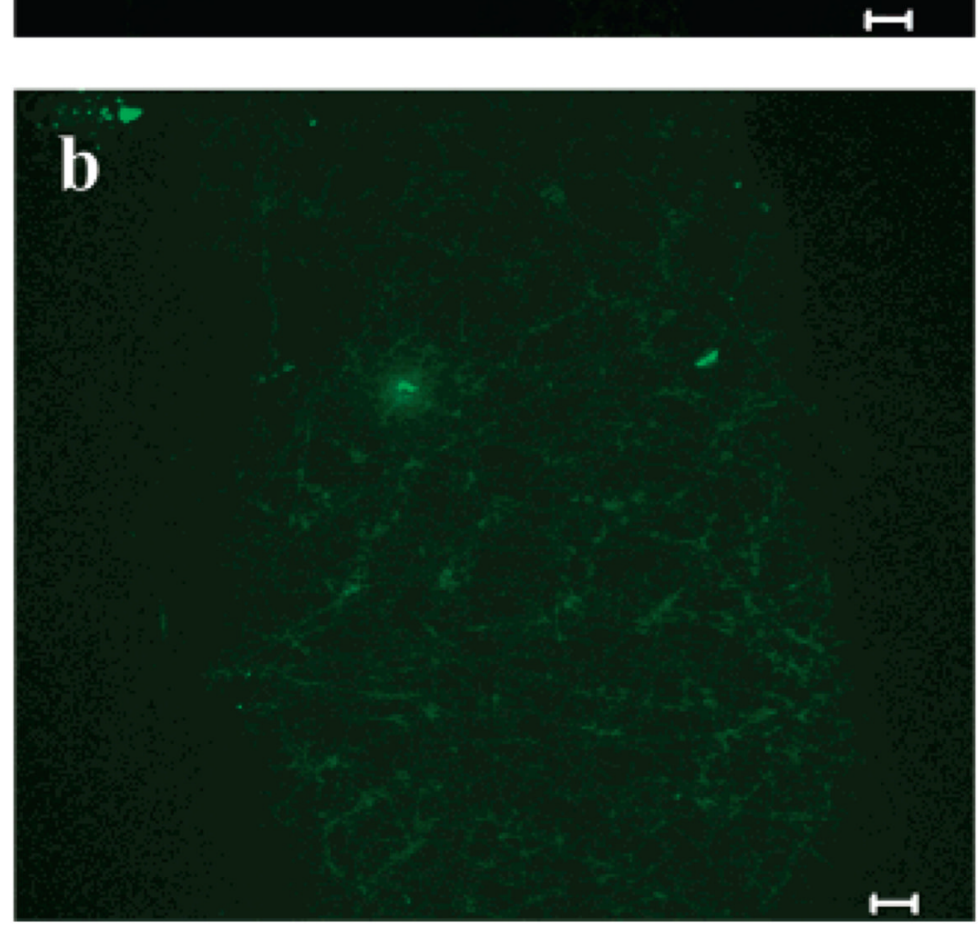

Figure 12.

Retention of heparin within electrospun matrices as observed by multiphoton microscopy for the (a) LMWH-dye/PLGA fibers and (b) PEG-LMWH-dye/PLGA fibers (10 $\mu \mathrm{m}$ scale bars). 


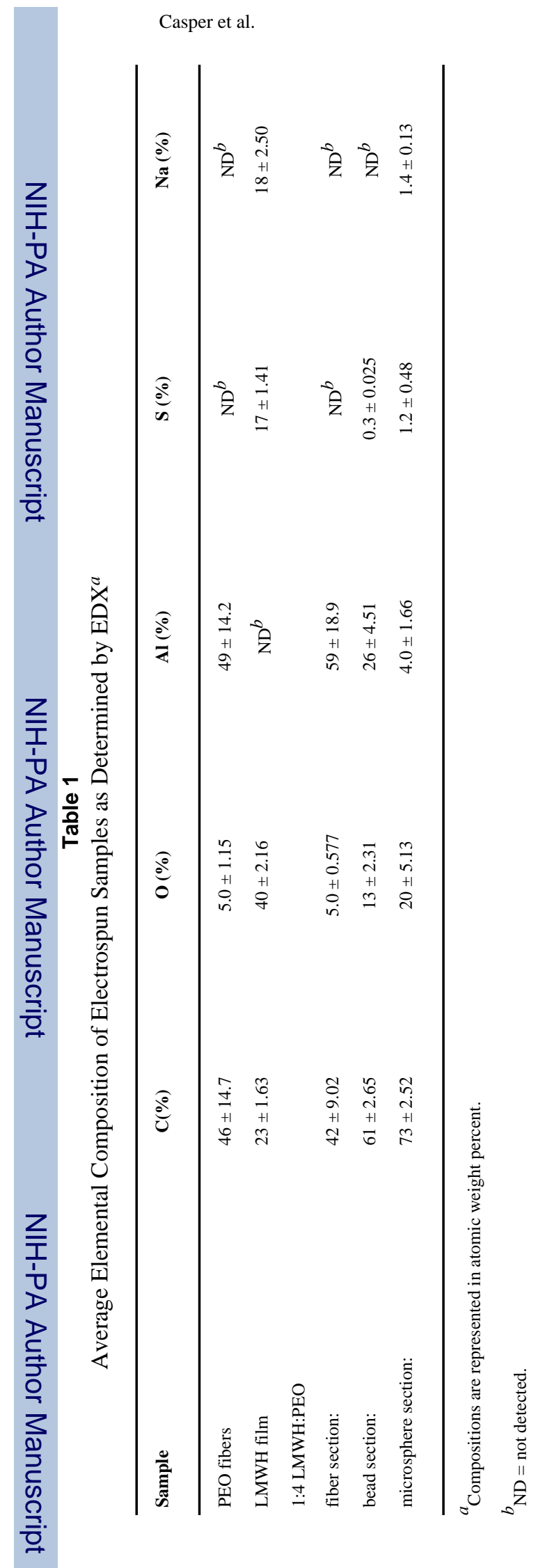

Biomacromolecules. Author manuscript; available in PMC 2009 September 15. 